\title{
Vanishing spin-Hall current in a diffusive Rashba two-dimensional electron system: A quantum Boltzmann equation approach
}

\author{
S. Y. Liu, ${ }^{1,2, \text { X X. L. Lei, }{ }^{1} \text { and Norman J. M. Horing }}{ }^{2}$ \\ ${ }^{1}$ Department of Physics, Shanghai Jiaotong University, 1954 Huashan Road, Shanghai 200030, China \\ ${ }^{2}$ Department of Physics and Engineering Physics, \\ Stevens Institute of Technology, Hoboken, New Jersey 07030, USA
}

(Dated: November 6, 2018)

\begin{abstract}
We present a quantum Boltzmann equation analysis of the spin-Hall effect in a diffusive Rashba two-dimensional electron system. Within the framework of the self-consistent Born approximation, we consider the roles of disorder-induced quasiclassical relaxation, collisional broadening of the quasiparticles, and the intracollisional field effect in regard to spin-Hall dynamics. We present an analytical proof that the spin-Hall current vanishes, independently of the coupling strength, of the quasiparticle broadening, of temperature and of the specific form of the isotropic scattering potential. A sum relation of the collision terms in a helicity basis is also examined.
\end{abstract}

PACS numbers: 72.10.-d, 72.25.Dc, 73.50.Bk

\section{INTRODUCTION}

The spin-Hall effect refers to the appearance of a net polarized spin flow along the direction perpendicular to an external applied dc electric field. This phenomenon suggests a possible mechanism to control spin dynamics using an electric field and may be employed to resolve the challenge of spin injection in the emerging field of spintronics. In early studies, the spin-Hall effect was associated with the disorder-induced spin-orbit (SO) interaction, of an extrinsic nature ${ }^{1.2}$ More recently, another disorder-free spin-Hall effect was predicted, respectively, in bulk $p$-doped semiconductors ${ }^{3}$ and in Rashba two-dimensional (2D) systems. ${ }^{4}$ This intrinsic effect essentially arises from disorderindependent SO interactions, such as the Rashba SO coupling, the SO interaction involved in the Luttinger model, etc. Experimentally, the spin-Hall effect has been observed in a bulk $n$-type semiconductor ${ }^{5}$ and in a $2 \mathrm{D}$ heavy-hole system ${ }^{6}$

In Rashba 2D electron systems, the spin-Hall current can be strongly affected by a spin-conserving interaction between electrons and impurities. This issue has been investigated in the diffusive regime extensively by various methods, including the Kubo formula, $\underset{7.89 .10 .11 .12}{1}$ the spin-density matrix method, 13 and a nonequilibrium Green's function approach ${ }^{14.15 .16}$ Physically, to examine the effect of disorder on spin-Hall current, we must simultaneously take into account collisional broadening and quasiclassical relaxation, as well as the intracollisional field effect $17,18,19$ produced by the action of the electric field during the course of electron-impurity scattering. The most important disorder effect on spin-Hall current is associated with quasiclassical relaxation. This scattering process yields an additional term to the spin-Hall current, causing it to vanish 9.10 .11 .12 .13 .14 .15 In addition, the collision-induced spread of the quasiparticle density of states (DOS)-namely, collisional broadening-also influences the spin-Hall effect. It reduces the value of the intrinsic spin-Hall conductivity with increasing broadening of the DOS $\stackrel{7.8}{=}$ For short-range disorder, the total spin-Hall current still vanishes when collisional broadening in both the intrinsic and quasiclassical terms of the spin-Hall current is considered 9.10 .11 .12 There is yet another disorder-induced quantum effect-namely,

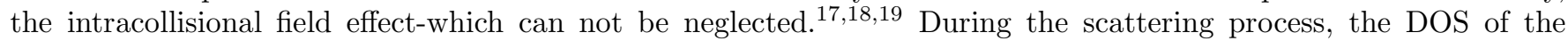
quasiparticles can be modified by the external electric field, whereupon relaxation becomes dependent on this field even in linear response. To date, only Sugimoto et al. have tried to investigate the spin-Hall effect including these

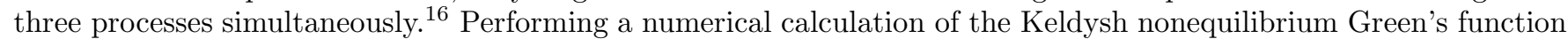
in a spin basis, they found a generally nonvanishing spin-Hall current: the value of the spin-Hall conductivity they obtained depends on the spin-orbit coupling constant, the transport lifetime, and the Fermi momentum.

In this paper, we analytically investigate the spin-Hall effect in Rashba 2D systems using the nonequilibrium Green's function method, essentially the same as that used by Sugimoto et al. 16 but in a helicity basis. We carefully analyze the nonequilibrium retarded and lesser Green's functions by simultaneously taking account of quasiclassical relaxation, collisional broadening, and the intracollisional field effect. We prove analytically that the spin-Hall current vanishes, irrespective of the spin-orbit coupling strength, of the form of the isotropic impurity potential, of temperature, and of the collisional broadening of the DOS. We also discuss a sum relation of the relaxation terms in the helicity basis.

This paper is organized as follows. In Sec. II, we present the kinetic equation for the "lesser" Green's function. The vanishing of spin-Hall current is proven in Sec. III. Finally, we review our results in Sec. IV. Several appendixes provide requisite details which could be read before Eq. (3) by readers desiring a "proof first" exposition. 


\section{KINETIC EQUATION FOR A 2D RASHBA SYSTEM}

We consider a 2D Rashba electron system with a single-particle noninteracting Hamiltonian given by̌ㅡㄹ

$$
\check{h}_{0}(\mathbf{p})=\frac{p^{2}}{2 m}+\alpha \mathbf{p} \cdot(\mathbf{n} \times \sigma) .
$$

Here, $\mathbf{p} \equiv\left(p_{x}, p_{y}\right) \equiv\left(p \cos \phi_{\mathbf{p}}, p \sin \phi_{\mathbf{p}}\right)$ is the $2 \mathrm{D}$ electron momentum, $m$ is the effective mass, $\sigma \equiv\left(\sigma_{x}, \sigma_{y}, \sigma_{z}\right)$ are the Pauli matrices, $\alpha$ is a spin-orbit coupling constant, and $\mathbf{n}$ is a unit vector perpendicular to the 2D electron plane. By a local unitary spinor transformation $U_{\mathbf{p}}$,

$$
U_{\mathbf{p}}=\frac{1}{\sqrt{2}}\left(\begin{array}{cc}
1 & 1 \\
i \mathrm{e}^{i \phi_{\mathbf{p}}} & -i \mathrm{e}^{i \phi_{\mathbf{p}}}
\end{array}\right)
$$

the Hamiltonian (11) can be diagonalized as $\hat{h}_{0}(p)=U_{\mathbf{p}}^{+} \breve{h}_{0}(\mathbf{p}) U_{\mathbf{p}}=\operatorname{diag}\left(\varepsilon_{1}(p), \varepsilon_{2}(p)\right)$ with $\varepsilon_{\mu}(p)=\frac{p^{2}}{2 m}+(-1)^{\mu} \alpha p$ $(\mu=1,2)$ as dispersion relations of two spin-orbit-coupled bands. In this paper, we investigate the spin-Hall effect in a 2D Rashba electron system driven by a constant, uniform electric field $\mathbf{E}$ along the $x$ axis. In the Coulomb gauge, the electric field can be described by a scalar potential, $V \equiv-e \mathbf{E} \cdot \mathbf{r}$, with $\mathbf{r}$ as the electron coordinate.

In a realistic $2 \mathrm{D}$ system, electrons experience scattering by impurities. We assume that the interaction between electrons and impurities can be characterized by an isotropic potential, $V(|\mathbf{p}-\mathbf{k}|)$, corresponding to scattering of an electron from state $\mathbf{p}$ to state $\mathbf{k}$.

The nonequilibrium Green's functions $\check{G}_{\mathbf{p}}^{r,<}$ in spin basis for 2D electron systems with Rashba SO coupling are defined as usual. They are $2 \times 2$ matrices and obey Dyson equations presented in Appendix A. For brevity, hereafter, we employ a subscript $\mathbf{p}$ to denote the arguments of the Green's functions and self-energies, $(\mathbf{p}, \omega)$. It is most convenient to study the lesser Green's function in the helicity basis, $\hat{\mathrm{G}}_{\mathbf{p}}^{<}=U_{\mathbf{p}}^{+} \check{\mathrm{G}}_{\mathbf{p}}^{<} U_{\mathbf{p}}$, in which the unperturbed lesser Green's function is diagonal. Using the transformation from spin basis to helicity basis, the Dyson equation for $\hat{G}_{\mathbf{p}}^{<}$ can be written as

$$
i e \mathbf{E} \cdot\left(\frac{\partial}{\partial \mathbf{p}}+\mathbf{p} \frac{\partial}{\partial \omega}\right) \hat{\mathrm{G}}_{\mathbf{p}}^{<}+i e \mathbf{E} \cdot\left[\hat{\mathrm{G}}_{\mathbf{p}}^{<}, \nabla_{\mathbf{p}} U_{\mathbf{p}}^{+} U_{\mathbf{p}}\right]+\alpha p\left[\sigma_{z}, \hat{\mathrm{G}}_{\mathbf{p}}^{<}\right]+\frac{i \alpha}{2} e \mathbf{E} \cdot \frac{\partial}{\partial \omega} \hat{\mathbf{B}}_{\mathbf{p}}^{<}=\hat{I}_{l},
$$

with $\hat{\mathbf{A}}_{\mathbf{p}}^{<}=\left[\mathbf{N}_{\mathbf{p}}, \hat{\mathrm{G}}_{\mathbf{p}}^{<}\right], \hat{\mathbf{B}}_{\mathbf{p}}^{<}=\left\{\mathbf{N}_{\mathbf{p}}, \hat{\mathrm{G}}_{\mathbf{p}}^{<}\right\}, \mathbf{N}_{\mathbf{p}}=U_{\mathbf{p}}^{+}(\mathbf{n} \times \sigma) U_{\mathbf{p}}$, and $\hat{I}_{l}$ as a scattering term. In general, $\hat{I}_{l}$ has a complicated form because the momentum and time variables of the Green's functions and self-energies are intertwined due to the presence of the electric field $\stackrel{22}{\underline{2}}$ Physically, this feature involves the intracollisional field effect $\frac{17.18 .19}{1}$ and results in an additional electric-field-dependent scattering. In this paper, we restrict our considerations to the linear response regime. Based on this, the scattering term can be expressed as the sum of three terms: $\hat{I}_{l}=\hat{I}_{l 1}+\hat{I}_{l 2}+\hat{I}_{l 3}$. The first term, $\hat{I}_{l 1}$ does not involve the explicit appearance of the electric field (although it is implicit in $\hat{\mathrm{G}}_{\mathbf{p}}^{r,<}$ and selfenergies $\hat{\Sigma}_{\mathbf{p}}^{r,<}$ ). The terms $\hat{I}_{l 2}$ and $\hat{I}_{l 3}$ arise from the first-order gradient expansion involving explicit linear dependence on the electric field, and they are a manifestation of the intracollisional field effect. The explicit forms of quantities $\hat{I}_{l 1}$ and $\hat{I}_{l 2}$ are similar to $\breve{I}_{l 1}$ and $\breve{I}_{l 2}$, respectively, but with helicity-basis Green's functions and self-energies in place of the corresponding spin-basis ones (the forms of $\check{I}_{l 1}$ and $\check{I}_{l 2}$ are provided in Appendix A). The term $\hat{I}_{l 3}$ arises from the local nature of the transformation and its form is obtained from $\hat{I}_{l 2}$ by using $\frac{i}{2} \nabla_{\mathbf{p}} \phi_{\mathbf{p}}\left[\cdots, \sigma_{x}\right]$ instead of operator $\nabla_{\mathbf{p}}$.

From the explicit form of the scattering term, it may be seen that $\hat{I}_{l}$ relates to the retarded Green's function $\hat{\mathrm{G}}_{\mathbf{p}}^{r}$ in which the collisional broadening is considered. Detailed properties of $\hat{G}_{\mathbf{p}}^{r}$ are analyzed in Appendix B. We find that the linear electric field correction to the retarded Green's function $\hat{\mathrm{G}}_{1 \mathrm{p}}^{r}$ in a 2D Rashba system does not vanish in general (although it has vanishing diagonal elements). Also, we make clear that disorder scattering is not essential for the nonvanishing of $\hat{\mathrm{G}}_{1 \mathbf{p}}^{r}$ : $\hat{\mathrm{G}}_{1 \mathbf{p}}^{r}$ exists even when the electron-impurity collisions are ignored.

It is well known that the linear electric field correction to the retarded Green's function vanishes for a one-band

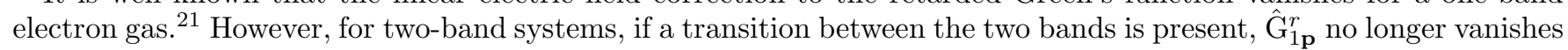
(see, for example, Ref.22 p. 215). In the 2D Rashba system that we study, there are two spin-orbit-coupled bands arising from the structure asymmetry and a polarization process can be induced between these two bands by a dc field ${ }^{23}$ Accordingly, the linear dc electric field correction to $\hat{\mathrm{G}}_{\mathbf{p}}^{r}$ in a 2D Rashba electron system is nonvanishing.

In Eq. (3), the electron-impurity scattering is embedded in the self-energies $\hat{\Sigma}_{\mathbf{p}}^{r,<}$. In the present paper, we consider the disorder collisions only in the self-consistent Born approximation. It is widely accepted that this treatment is 
sufficiently accurate to analyze transport properties in the diffusive regime. On this basis, the self-energies in helicity basis take the forms

$$
\hat{\Sigma}_{\mathbf{p}}^{r,<}=n_{i} \sum_{\mathbf{k}} \hat{T}(\mathbf{p}, \mathbf{k}) \hat{\mathrm{G}}_{\mathbf{k}}^{r,<} \hat{T}^{+}(\mathbf{p}, \mathbf{k})
$$

where $\hat{T}(\mathbf{p}, \mathbf{k})=U^{+}(\mathbf{k}) V(\mathbf{p}-\mathbf{k}) U(\mathbf{p})$ and $n_{i}$ is the impurity density.

Further, we assume that the applied dc field is weak enough that only the linear response is required to be considered. After linearizing Eq. (3), we can write the kinetic equation for the linear electric field correction to the lesser Green's function, $\hat{\mathrm{G}}_{1 \mathbf{p}}^{<}=\hat{\mathrm{G}}_{\mathbf{p}}^{<}-\hat{\mathrm{G}}_{0 \mathbf{p}}^{<}$, as

$$
-\alpha p \hat{C}_{1}+i e \mathbf{E} \cdot \nabla_{\mathbf{p}} \hat{\mathrm{G}}_{0 \mathbf{p}}^{<}-\frac{1}{2} e \mathrm{E} \cdot \nabla_{\mathbf{p}} \phi_{\mathbf{p}} \hat{D}_{0}+R=\hat{I}_{l}^{(1)}
$$

where the matrices $\hat{C}_{1}$ and $\hat{D}_{0}$ are given by

$$
\begin{gathered}
\hat{C}_{1}=\left(\begin{array}{cc}
0 & -2\left(\hat{\mathrm{G}}_{1 \mathbf{p}}^{<}\right)_{12} \\
2\left(\hat{\mathrm{G}}_{1 \mathbf{p}}^{<}\right)_{21} & 0
\end{array}\right), \\
\hat{D}_{0}=\left(\begin{array}{cc}
0 & \left(\hat{\mathrm{G}}_{0 \mathbf{p}}^{<}\right)_{11}-\left(\hat{\mathrm{G}}_{0 \mathbf{p}}^{<}\right)_{22} \\
\left(\hat{\mathrm{G}}_{0 \mathbf{p}}^{<}\right)_{22}-\left(\hat{\mathrm{G}}_{0 \mathbf{p}}^{<}\right)_{11} & 0
\end{array}\right) .
\end{gathered}
$$

$R$ is a remainder term which can be expressed as a total derivative with respect to $\omega$. $\hat{\mathrm{G}}_{0 \mathbf{p}}^{<}$is the unperturbed equilibrium lesser Green's function. It is a diagonal matrix and simply relates to the retarded $\hat{\mathrm{G}}_{0 \mathbf{p}}^{r}$ by the KuboMartin-Schwinger relation 24

$$
\hat{\mathrm{G}}_{0 \mathbf{p}}^{<}=-2 i n_{\mathrm{F}}(\omega) \operatorname{Im} \hat{\mathrm{G}}_{0 \mathbf{p}}^{r}
$$

with $n_{\mathrm{F}}(\omega)$ as the Fermi function.

The scattering term $\hat{I}_{l}^{(1)}$ is the sum of $\hat{I}_{l 1}^{(1)}, \hat{I}_{l 2}^{(1)}$, and $\hat{I}_{l 3}^{(1)}$, the linear electric field parts of $\hat{I}_{l 1}, \hat{I}_{l 2}$ and $\hat{I}_{l 3}$. The explicit forms of these quantities are presented in Appendix C. It should be noted that $\hat{I}_{l 2}^{(1)}$ and $I_{l 3}^{(1)}$ are diagonal and off-diagonal matrices, respectively. In deriving Eq. (5), the properties of the retarded Green's function in the presence of collisional broadening are employed: its linear electric field correction is an off-diagonal matrix, while the unperturbed one is diagonal.

From Eq. (5) we can formally express the off-diagonal element of $\hat{\mathrm{G}}_{1 \mathrm{p}}^{<}$as

$$
\begin{aligned}
\left(\hat{\mathrm{G}}_{1 \mathbf{p}}^{<}\right)_{12}= & \frac{1}{2 \alpha p}\left\{\frac{1}{2} e \mathbf{E} \cdot \nabla_{\mathbf{p}} \phi_{\mathbf{p}}\left[\left(\hat{\mathrm{G}}_{0 \mathbf{p}}^{<}\right)_{11}-\left(\hat{\mathrm{G}}_{0 \mathbf{p}}^{<}\right)_{22}\right]\right. \\
& \left.+\left(I_{l}^{(1)}\right)_{12}-R_{12}\right\}
\end{aligned}
$$

At the same time, the diagonal elements of $I_{l}^{(1)}$ take the form,

$$
\left(I_{l}^{(1)}\right)_{\mu \mu}=i e \mathbf{E} \cdot \nabla_{\mathbf{p}}\left(\hat{\mathrm{G}}_{0 \mathbf{p}}^{<}\right)_{\mu \mu}+R_{\mu \mu},
$$

with $\mu=1,2$.

\section{VANISHING SPIN-HALL CURRENT}

We are interested in a spin current polarized along the $z$-direction and flowing along the $y$ axis when a dc electric field $\mathbf{E}$ is applied along the $x$ axis-i.e. $J_{y}^{z}$. In spin basis, it relates to the single-particle spin current operator $\check{j}_{y}^{z}$ by

$$
J_{y}^{z}=\sum_{\mathbf{p}} \int \frac{d \omega}{2 \pi} \operatorname{Tr}\left[\check{j}_{y}^{z} \operatorname{Im}\left(\check{\mathrm{G}}_{1 \mathbf{p}}^{<}\right)\right] .
$$




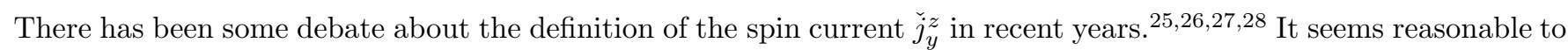
define the spin current from a continuity equation relating to the spin, as shown in Ref.28. Conventionally, the singleparticle spin current in spin basis is defined as $\check{j}_{y}^{z}=\frac{1}{4}\left\{v_{y}, \sigma_{z}\right\}$ with the velocity operator $v_{y}$. Zhang et al. suggested that the spin current operator has an additional term arising from the spin torque density, $\left(\check{j}_{y}^{z}\right)_{\text {add }}=(y / 2 i)\left[\sigma_{z}, \check{h}_{0}(\mathbf{p})\right] \mathrm{s}^{28}$ Actually, the contribution from this term to spin-Hall current vanishes in a homogeneous 2D Rashba electron system to linear order in the dc field. This can be seen from the fact that the additional spin-Hall current operator, explicitly given by $\left(\check{j}_{y}^{z}\right)_{\text {add }}=\alpha y \mathbf{p} \cdot \sigma=-i \alpha \frac{\partial}{\partial p_{y}} \mathbf{p} \cdot \sigma$, is invariant under inversion of momentum $\mathbf{p}, i . e . \mathbf{p} \rightarrow-\mathbf{p}$. However, $\mathbf{p}$ reversal leads to a sign change of the linear electric field correction to the lesser Green's function: $\hat{\mathrm{G}}_{1,-\mathbf{p}}^{<}=-\hat{\mathrm{G}}_{1 \mathbf{p}}^{<}$ [this result can be seen from Eq. (5) by inverting the momentum]. Based on this, it follows from the $\mathbf{p}$ integration of Eq. (11) that the contribution to spin-Hall current arising from the additional spin-current term vanishes. Hence, we only need to consider the conventional component of the spin-Hall current operator.

Consequently, we write the spin current operator $J_{y}^{z}$ in the simple helicity-basis description as 15

$$
J_{y}^{z}=\sum_{\mathbf{p}} \int \frac{d \omega}{2 \pi} \frac{p_{y}}{m} \operatorname{Im}\left(\hat{\mathbf{G}}_{\mathbf{p}}^{<}\right)_{12}
$$

Substituting Eq. (9) into this equation, we obtain

$$
J_{y}^{z}=\sum_{\mathbf{p}} \int \frac{d \omega}{2 \pi} \frac{1}{4 m \alpha} \sin \phi_{\mathbf{p}} e \mathbf{E} \cdot \nabla_{\mathbf{p}} \phi_{\mathbf{p}} \operatorname{Im}\left[\left(\hat{\mathrm{G}}_{0 \mathbf{p}}^{<}\right)_{11}-\left(\hat{\mathrm{G}}_{0 \mathbf{p}}^{<}\right)_{22}\right]+\sum_{\mathbf{p}} \int \frac{d \omega}{2 \pi} \frac{1}{2 m \alpha} \sin \phi_{\mathbf{p}} \operatorname{Im}\left(I_{l}^{(1)}\right)_{12} .
$$

In this, the $\omega$ integration over the term $R$ vanishes.

To further simplify the expression for $J_{y}^{z}$, we analyze the second term on the right-hand side of Eq. (13). In connection with this, we find that there is a sum relation among the elements of $I_{l}^{(1)}$ in the self-consistent Born approximation:

$$
\sum_{\mathbf{p}} \int \frac{d \omega}{2 \pi} \sin \phi_{\mathbf{p}} \operatorname{Im}\left(I_{l}^{(1)}\right)_{12}=\sum_{\mathbf{p}} \int \frac{d \omega}{2 \pi} \frac{\cos \phi_{\mathbf{p}}}{2}\left[\operatorname{Re}\left(I_{l}^{(1)}\right)_{11}-\operatorname{Re}\left(I_{l}^{(1)}\right)_{22}\right]
$$

Furthermore, separate relations also hold for each component of $I_{l}^{(1)}$ :

$$
\begin{aligned}
& \sum_{\mathbf{p}} \int \frac{d \omega}{2 \pi} \sin \phi_{\mathbf{p}} \operatorname{Im}\left(I_{l 1}^{(1)}\right)_{12}=\sum_{\mathbf{p}} \int \frac{d \omega}{2 \pi} \frac{\cos \phi_{\mathbf{p}}}{2}\left[\operatorname{Re}\left(I_{l 1}^{(1)}\right)_{11}-\operatorname{Re}\left(I_{l 1}^{(1)}\right)_{22}\right], \\
& \sum_{\mathbf{p}} \int \frac{d \omega}{2 \pi} \sin \phi_{\mathbf{p}} \operatorname{Im}\left(I_{l 3}^{(1)}\right)_{12}=\sum_{\mathbf{p}} \int \frac{d \omega}{2 \pi} \frac{\cos \phi_{\mathbf{p}}}{2}\left[\operatorname{Re}\left(I_{l 2}^{(1)}\right)_{11}-\operatorname{Re}\left(I_{l 2}^{(1)}\right)_{22}\right] .
\end{aligned}
$$

The detailed proof of these equations is presented in Appendix D.

Based on the sum relation Eqs. (14) and (10) for diagonal components of $I_{l}^{(1)}$, we obtain

$$
\begin{aligned}
J_{y}^{z}= & \sum_{\mathbf{p}} \int \frac{d \omega}{2 \pi} \frac{\sin \phi_{\mathbf{p}}}{4 m \alpha} e \mathbf{E} \cdot \nabla_{\mathbf{p}} \phi_{\mathbf{p}} \operatorname{Im}\left[\left(\hat{\mathrm{G}}_{0 \mathbf{p}}^{<}\right)_{11}-\left(\hat{\mathrm{G}}_{0 \mathbf{p}}^{<}\right)_{22}\right] \\
& -\sum_{\mathbf{p}} \int \frac{d \omega}{2 \pi} \frac{\cos \phi_{\mathbf{p}}}{4 m \alpha} e \mathbf{E} \cdot\left(\nabla_{\mathbf{p}} \operatorname{Im}\left[\left(\hat{\mathrm{G}}_{0 \mathbf{p}}^{<}\right)_{11}-\left(\hat{\mathrm{G}}_{0 \mathbf{p}}^{<}\right)_{22}\right]\right) \\
= & -\sum_{\mathbf{p}} \int \frac{d \omega}{2 \pi} \frac{e \mathbf{E} \cdot \nabla_{\mathbf{p}} \cos \phi_{\mathbf{p}}}{4 m \alpha} \operatorname{Im}\left[\left(\hat{\mathrm{G}}_{0 \mathbf{p}}^{<}\right)_{11}-\left(\hat{\mathrm{G}}_{0 \mathbf{p}}^{<}\right)_{22}\right] \\
& -\sum_{\mathbf{p}} \int \frac{d \omega}{2 \pi} \frac{\cos \phi_{\mathbf{p}}}{4 m \alpha} e \mathbf{E} \cdot\left(\nabla_{\mathbf{p}} \operatorname{Im}\left[\left(\hat{\mathrm{G}}_{0 \mathbf{p}}^{<}\right)_{11}-\left(\hat{\mathrm{G}}_{0 \mathbf{p}}^{<}\right)_{22}\right]\right) .
\end{aligned}
$$

It is apparent from Eq. (17) that the integrand on its right-hand side becomes a total derivative with respect to p. Consequently, the vanishing of the spin-Hall current is obtained immediately upon the momentum integration. We note that this analytical result is significantly different from that of the numerical analysis of Sugimato et al., 16 which 
purports to exhibit a nonvanishing spin-Hall current. Our analysis shows that this is not the case even with the "new" spin current term included.

It is obvious from Eq. (17) that the spin-Hall current vanishes independently of the strength of SO coupling, of the specific form of the isotropic scattering potential, and of the lattice temperature. Moreover, it is valid even for arbitrary broadening of the DOS. However, this result holds only in the diffusive regimes-namely, in the regime $l_{D} k_{\mathrm{F}}>1\left(l_{D}\right.$ is diffusion length and $k_{\mathrm{F}}$ is the Fermi wave vector), because we restricted treatment of the electronimpurity scattering to the self-consistent Born approximation (otherwise, contributions from the maximally crossed diagrams of electron-impurity scattering would have to be taken into account).

\section{CONCLUSIONS}

The spin-Hall effect in 2D diffusive Rashba systems has been investigated by means of a quantum Boltzmann approach in this paper. In the self-consistent Born approximation, we have considered all effects induced by electronimpurity scattering-namely, quasiclassical relaxation, collisional broadening, and the intracollisional field effect. We have proven analytically that the spin-Hall current vanishes, irrespective of the strength of SO coupling, of the broadening of DOS, and of the form of the isotropic scattering potential. The sum relation of the scattering terms in the helicity basis has been analyzed.

Our treatment is applicable at nonvanishing temperatures (not restricted to $T=0$ ), and it is not restricted in anyway to short-range scattering potentials. We have also made it clear that the spin-Hall effect vanishes even when correctly considering the influence of the electric field in the scattering process (intracollisional field effect).

It should be noted that this proof of vanishing of spin-Hall current also applies for a Dresselhaus linear-momentum SO coupling, since it relates simply to the Rashba SO interaction by a global transformation. Furthermore, we have also examined the spin-Hall effect when both the Rashba and Dresselhaus SO interactions are active, again finding zero spin-Hall current.

Nevertheless, we note that our proof of vanishing of the spin-Hall effect has its validity limited to $2 \mathrm{D}$ systems with linear-momentum SO coupling. Also, this proof requires $\breve{h}_{0}$ to be parabolic in the absence of SO coupling. Otherwise, the spin-Hall effect should be examined anew. This is to say that our result does not conflict with the nonzero experimental spin-Hall observations ${ }^{5.6}$ : the Hamiltonians of bulk and 2D heavy-hole systems in which nonvanishing spin-Hall currents were observed differ significantly from the Hamiltonian $\check{h}_{0}(\mathbf{p})$ studied in this paper.

Note added. After this work was completed and submitted, an unpublished paper of Krotkov and Das Sarma reported a nonvanishing spin-Hall effect in 2D Rashba electron systems by considering a nonparabolic effect 29

\section{Acknowledgments}

One of the authors (SYL) would like to gratefully acknowledge the invaluable discussions with Dr. M. W. Wu, Dr. W. Xu, Dr. W. S. Liu, and Dr. Y. Chen. This work was supported by projects of the National Science Foundation of China and the Shanghai Municipal Commission of Science and Technology and by the Youth Scientific Research Startup Founds of SJTU. N.J.M.H. is supported by the Department of Defense through the DURINT program administered by the U.S. Army Research Office, DAAD Grant No. 19-01-1-0592.

\section{APPENDIX A: NONEQUILIBRIUM GREEN'S FUNCTIONS IN SPIN BASIS}

In spin space, the $2 \times 2$ nonequilibrium Green's functions $\breve{G}\left(\mathbf{r}_{1}, \tau_{1} ; \mathbf{r}_{2}, \tau_{2}\right)$ obey Dyson equations given by ${ }^{22}$

$$
\begin{gathered}
\left\{i \frac{\partial}{\partial \tau_{1}}+\frac{1}{2 m} \vec{\nabla}_{\mathbf{r}_{1}}^{2}+i \alpha \nabla_{\mathbf{r}_{1}} \cdot(\mathbf{n} \times \sigma)+e \mathbf{E} \cdot \mathbf{r}_{1}\right\} \check{\mathrm{G}}\left(\mathbf{r}_{1}, \tau_{1} ; \mathbf{r}_{2}, \tau_{2}\right)= \\
\delta(1-2)+\int_{C} d \tau^{\prime} \int d \mathbf{r}^{\prime} \check{\Sigma}\left(\mathbf{r}_{1}, \tau_{1} ; \mathbf{r}^{\prime}, \tau^{\prime}\right) \check{\mathrm{G}}\left(\mathbf{r}^{\prime}, \tau^{\prime} ; \mathbf{r}_{2}, \tau_{2}\right), \\
\check{\mathrm{G}}\left(\mathbf{r}_{1}, \tau_{1} ; \mathbf{r}_{2}, \tau_{2}\right)\left\{-i \frac{\partial}{\partial \tau_{2}}+\frac{1}{2 m} \vec{\nabla}_{\mathbf{r}_{2}}^{2}-i \alpha \nabla_{\mathbf{r}_{2}} \cdot(\mathbf{n} \times \sigma)+e \mathbf{E} \cdot \mathbf{r}_{2}\right\}=
\end{gathered}
$$




$$
\delta(1-2)+\int_{C} d \tau^{\prime} \int d \mathbf{r}^{\prime} \check{\mathrm{G}}\left(\mathbf{r}_{1}, \tau_{1} ; \mathbf{r}^{\prime}, \tau^{\prime}\right) \check{\Sigma}\left(\mathbf{r}^{\prime}, \tau^{\prime} ; \mathbf{r}_{2}, \tau_{2}\right) .
$$

In these equations, the electron-impurity interaction is embedded in the self-energies, $\check{\Sigma}\left(\mathbf{r}^{\prime}, \tau^{\prime} ; \mathbf{r}_{2}, \tau_{2}\right)$.

To further simplify Eqs. (A1) and (A2), we introduce relative and center-of-mass variables $\mathbf{r}=\mathbf{r}_{1}-\mathbf{r}_{2}, \tau=\tau_{1}-\tau_{2}$, $\mathbf{R}=\left(\mathbf{r}_{1}+\mathbf{r}_{2}\right) / 2$, and $T=\left(\tau_{1}+\tau_{2}\right) / 2$. Following this, we construct gauge-invariant retarded and lesser Green's functions $\breve{G}_{\mathbf{p}}^{r,<}$ in momentum-frequency space following established procedures ${ }^{22}$ Under homogeneous and steadystate conditions, these Green's functions satisfy the following equations:

$$
\begin{gathered}
2\left[\omega-\frac{p^{2}}{2 m}\right] \check{\mathbf{G}}_{\mathbf{p}}^{r}+i \alpha\left[i \mathbf{p} \cdot \check{\mathbf{B}}^{r}+\frac{1}{2} e \mathbf{E} \cdot \frac{\partial}{\partial \omega} \check{\mathbf{A}}_{\mathbf{p}}^{r}\right]=2+\check{I}_{r 1}+\check{I}_{r 2}, \\
i\left[e \mathbf{E} \cdot\left(\nabla_{\mathbf{p}}+\mathbf{p} \frac{\partial}{\partial \omega}\right)\right] \check{\mathbf{G}}_{\mathbf{p}}^{<}+i \alpha\left[i \mathbf{p} \cdot \check{\mathbf{A}}^{<}+\frac{1}{2} e \mathbf{E} \cdot \frac{\partial}{\partial \omega} \check{\mathbf{B}}_{\mathbf{p}}^{<}\right]=\check{I}_{l 1}+\check{I}_{l 2},
\end{gathered}
$$

where $\check{\mathbf{A}}_{\mathbf{p}}^{r,<} \equiv\left[\mathbf{n} \times \sigma, \check{G}_{\mathbf{p}}^{r,<}\right]$ and $\check{\mathbf{B}}_{\mathbf{p}}^{r,<} \equiv\left\{\mathbf{n} \times \sigma, \check{\mathrm{G}}_{\mathbf{p}}^{r,<}\right\}$ arise from the Rashba spin-orbit interaction term in the Hamiltonian. The first terms $\check{I}_{r 1}$ and $\check{I}_{l 1}$ on the right-hand sides of Eqs. (A3) and (A4), respectively, do not involve the explicit appearance of the electric field

$$
\begin{gathered}
\check{I}_{r 1}=\check{\Sigma}_{\mathbf{p}}^{r} \check{\mathrm{G}}_{\mathbf{p}}^{r}+\check{\mathrm{G}}_{\mathbf{p}}^{r} \check{\Sigma}_{\mathbf{p}}^{r}, \\
\check{I}_{l 1}=\check{\Sigma}_{\mathbf{p}}^{r} \check{\mathrm{G}}_{\mathbf{p}}^{<}-\check{\mathrm{G}}_{\mathbf{p}}^{<} \check{\Sigma}_{\mathbf{p}}^{a}-\check{\mathrm{G}}_{\mathbf{p}}^{r} \check{\Sigma}_{\mathbf{p}}^{<}+\check{\Sigma}_{\mathbf{p}}^{<} \check{\mathrm{G}}_{\mathbf{p}}^{a} .
\end{gathered}
$$

The terms $\check{I}_{r 2}$ and $\check{I}_{l 2}$ arise from the first-order gradient expansion involving explicit linear dependence on the electric field,

$$
\begin{gathered}
\check{I}_{r 2}=\frac{i}{2} e \mathbf{E} \cdot\left(\nabla_{\mathbf{p}} \check{\Sigma}_{\mathbf{p}}^{r} \frac{\partial}{\partial \omega} \check{G}_{\mathbf{p}}^{r}-\frac{\partial}{\partial \omega} \check{\Sigma}_{\mathbf{p}}^{r} \nabla_{\mathbf{p}} \check{\mathrm{G}}_{\mathbf{p}}^{r}\right. \\
\left.+\nabla_{\mathbf{p}} \check{\mathrm{G}}_{\mathbf{p}}^{r} \frac{\partial}{\partial \omega} \check{\Sigma}_{\mathbf{p}}^{r}-\frac{\partial}{\partial \omega} \check{G}_{\mathbf{p}}^{r} \nabla_{\mathbf{p}} \check{\Sigma}_{\mathbf{p}}^{r}\right), \\
\check{I}_{l 2}=\frac{i}{2} e \mathbf{E} \cdot\left(\nabla_{\mathbf{p}} \check{\Sigma}_{\mathbf{p}}^{r} \frac{\partial}{\partial \omega} \check{G}_{\mathbf{p}}^{<}-\frac{\partial}{\partial \omega} \check{\Sigma}_{\mathbf{p}}^{r} \nabla_{\mathbf{p}} \check{G}_{\mathbf{p}}^{<}\right. \\
-\nabla_{\mathbf{p}} \check{G}_{\mathbf{p}}^{<} \frac{\partial}{\partial \omega} \check{\Sigma}_{\mathbf{p}}^{a}+\frac{\partial}{\partial \omega} \check{G}_{\mathbf{p}}^{<} \nabla_{\mathbf{p}} \check{\Sigma}_{\mathbf{p}}^{a}-\nabla_{\mathbf{p}} \check{G}_{\mathbf{p}}^{r} \frac{\partial}{\partial \omega} \check{\Sigma}_{\mathbf{p}}^{<} \\
\left.+\frac{\partial}{\partial \omega} \check{G}_{\mathbf{p}}^{r} \nabla_{\mathbf{p}} \check{\Sigma}_{\mathbf{p}}^{<}+\nabla_{\mathbf{p}} \check{\Sigma}_{\mathbf{p}}^{<} \frac{\partial}{\partial \omega} \check{G}_{\mathbf{p}}^{a}-\frac{\partial}{\partial \omega} \check{\Sigma}_{\mathbf{p}}^{<} \nabla_{\mathbf{p}} \check{G}_{\mathbf{p}}^{a}\right) .
\end{gathered}
$$

Incidentally, ignoring the collisional broadening, i.e., the terms involving $\partial / \partial \omega$, and employing the generalized Kadanoff-Baym ansatz, Eq. A3 reduces to the kinetic equations of the distribution functions presented in the previous studies $\underline{30}$

\section{APPENDIX B: RETARDED GREEN'S FUNCTION}

To analyze the properties of the retarded Green's function, we begin from the Dyson equation in the helicity basis:

$$
2\left[\omega-\frac{p^{2}}{2 m}\right] \hat{\mathrm{G}}_{\mathbf{p}}^{r}+\alpha p\left\{\sigma_{z}, \hat{\mathrm{G}}_{\mathbf{p}}^{r}\right\}+\frac{i \alpha}{2} e \mathbf{E} \cdot \frac{\partial}{\partial \omega} \hat{\mathbf{A}}_{\mathbf{p}}^{r}=2+\hat{I}_{r 1}+\hat{I}_{r 2}+\hat{I}_{r 3} .
$$

This equation is obtained from Eq. A3 by the transformation from spin basis to the helicity basis. The quantities $\hat{I}_{r 1}$ and $\hat{I}_{r 2}$ are the helicity-basis analogues of the quantities $\check{I}_{r 1}$ and $\check{I}_{r 2}$, respectively. $\hat{I}_{r 3}$ has a form similar to $\hat{I}_{r 2}$, but with $\frac{i}{2} \nabla_{\mathbf{p}} \phi_{\mathbf{p}}\left[\cdots, \sigma_{x}\right]$ in place of the operator $\nabla_{\mathbf{p}}$. 
Obviously, in the helicity basis, the noninteracting retarded Green's function $\hat{g}_{\mathbf{p}}^{r}$ is diagonal

$$
\hat{g}_{\mathbf{p}}^{r}=\operatorname{diag}\left(\left(\omega-\varepsilon_{1}(p)+i \delta\right)^{-1},\left(\omega-\varepsilon_{2}(p)+i \delta\right)^{-1}\right) .
$$

Including collisional broadening in the definition of the unperturbed retarded Green's function $\hat{\mathrm{G}}_{0 \mathbf{p}}^{r}$, it obeys the Dyson equation in the absence of the electric field, as

$$
\left[\omega-\frac{p^{2}}{2 m}\right] \hat{\mathrm{G}}_{0 \mathbf{p}}^{r}+\frac{\alpha p}{2}\left\{\sigma_{z}, \hat{\mathrm{G}}_{0 \mathbf{p}}^{r}\right\}=1+\frac{1}{2}\left(\hat{\Sigma}_{0 \mathbf{p}}^{r} \hat{\mathrm{G}}_{0 \mathbf{p}}^{r}+\hat{\mathrm{G}}_{0 \mathbf{p}}^{r} \hat{\Sigma}_{0 \mathbf{p}}^{r}\right) .
$$

The solution of this equation is also a diagonal matrix, $\left(\hat{\mathrm{G}}_{0 \mathbf{p}}^{r}\right)_{12}=\left(\hat{\mathrm{G}}_{0 \mathbf{p}}^{r}\right)_{21}=0$, with elements independent of the direction of momentum. To verify this, we examine the form of the self-energy $\hat{\Sigma}_{0 \mathbf{p}}^{r}$ below.

Let us first discuss the forms of self-energies $\hat{\Sigma}_{\mathbf{p}}^{r,<}$ in general. Installing the actual form of matrix $U_{\mathbf{p}}$ into Eq. (4), we find

$$
\begin{aligned}
\hat{\Sigma}_{\mathbf{p}}^{r,<}= & \frac{1}{2} n_{i} \sum_{\mathbf{k}}|V(\mathbf{p}-\mathbf{k})|^{2}\left\{a_{1} \hat{\mathrm{G}}_{\mathbf{k}}^{r,<}\right. \\
& \left.+a_{2} \hat{\sigma}_{x} \hat{\mathrm{G}}_{\mathbf{k}}^{r,<} \hat{\sigma}_{x}+i a_{3}\left[\hat{\sigma}_{x}, \hat{\mathrm{G}}_{\mathbf{k}}^{r,<}\right]\right\} .
\end{aligned}
$$

Explicitly, the self-energies can also be rewritten as

$$
\begin{array}{r}
\left(\hat{\Sigma}_{\mathbf{p}}^{r,<}\right)_{\mu \nu}=\frac{n_{i}}{2} \sum_{\mathbf{k}}|V(\mathbf{p}-\mathbf{k})|^{2}\left\{a_{1}\left(\hat{\mathrm{G}}_{\mathbf{k}}^{r,<}\right)_{\mu \nu}+\right. \\
\left.a_{2}\left(\hat{\mathrm{G}}_{\mathbf{k}}^{r,<}\right)_{\bar{\mu} \bar{\nu}}+i a_{3}\left[\left(\hat{\mathrm{G}}_{\mathbf{k}}^{r,<}\right)_{\bar{\mu} \nu}-\left(\hat{\mathrm{G}}_{\mathbf{k}}^{r,<}\right)_{\mu \bar{\nu}}\right]\right\} .
\end{array}
$$

In these expressions, $\bar{\mu}=3-\mu$ and $a_{i}(i=1,2,3)$ are factors associated with the directions of momenta, $a_{1}=$ $1+\cos \left(\phi_{\mathbf{p}}-\phi_{\mathbf{k}}\right), a_{2}=1-\cos \left(\phi_{\mathbf{p}}-\phi_{\mathbf{k}}\right)$, and $a_{3}=\sin \left(\phi_{\mathbf{p}}-\phi_{\mathbf{k}}\right)$.

From Eq. (B5), we see that the diagonal elements of the Green's functions appearing in nondiagonal elements of $\hat{\Sigma}_{\mathbf{p}}^{r,<}$ are always combined with the factor $a_{3}$. If the solution of Eq. (B3), $\hat{\mathrm{G}}_{0 \mathbf{p}}^{r}$, is diagonal and independent of the direction of momentum, the self-energy term involving $a_{3}$ vanishes under momentum integration. Hence, the self-energy, $\hat{\Sigma}_{0 \mathbf{p}}^{r}$, also becomes diagonal and independent of momentum direction. As a result, Eq. (B3) reduces to a momentum-direction-independent equation for the diagonal elements of $\hat{\mathrm{G}}_{0 \mathbf{p}}^{r}$. This implies that the assumed form of $\hat{\mathrm{G}}_{0 \mathbf{p}}^{r}$ is consistent with Eq. (B3). From uniqueness of the solution of the Dyson equation we immediately conclude that such a $\hat{G}_{0 \mathbf{p}}^{r}$ is just the needed solution. It should be noted that, physically, the momentum-direction independence of the unperturbed retarded Green's function is associated with the rotational symmetry of the unperturbed Hamiltonian in helicity basis.

Considering the angular independence of $\hat{\mathrm{G}}_{0 \mathbf{p}}^{r}$, an important relation can be derived:

$$
\sum_{\mathbf{k}}|V(\mathbf{p}-\mathbf{k})|^{2} \sin \left(\phi_{\mathbf{p}}-\phi_{\mathbf{k}}\right)\left[\left(\hat{\mathrm{G}}_{0 \mathbf{k}}^{r}\right)_{22}-\left(\hat{\mathrm{G}}_{0 \mathbf{k}}^{r}\right)_{11}\right]=0 .
$$

This equation is needed to prove the sum relation of the scattering term (Appendix D).

To the first order in the dc field, the Dyson equation for the linear electric field correction to the retarded Green's function, $\hat{\mathrm{G}}_{1 \mathbf{p}}^{r}=\hat{\mathrm{G}}_{\mathbf{p}}^{r}-\hat{\mathrm{G}}_{0 \mathbf{p}}^{r}$, can be written as

$$
\begin{array}{r}
2\left[\omega-\frac{p^{2}}{2 m}+(-1)^{\mu} \delta_{\mu \nu} \alpha p\right]\left(\hat{\mathrm{G}}_{1}^{r}\right)_{\mu \nu}+\left(1-\delta_{\mu \nu}\right) \frac{\alpha e E}{2} \sin \phi_{\mathbf{p}} \frac{\partial}{\partial \omega}\left[\left(\hat{\mathrm{G}}_{0}^{r}\right)_{11}-\left(\hat{\mathrm{G}}_{0}^{r}\right)_{22}\right] \\
=\left(\hat{I}_{r 1}^{(1)}\right)_{\mu \nu}+\left(\hat{I}_{r 2}^{(1)}\right)_{\mu \nu}+\left(\hat{I}_{r 3}^{(1)}\right)_{\mu \nu}
\end{array}
$$

$\hat{I}_{r 1}^{(1)}, \hat{I}_{r 2}^{(1)}$, and $\hat{I}_{r 3}^{(1)}$ are the linear electric field parts of $\hat{I}_{r 1}, \hat{I}_{r 2}$ and $\hat{I}_{r 3} \cdot \hat{I}_{r 1}^{(1)}$ has the form

$$
\hat{I}_{r 1}^{(1)}=2\left(\begin{array}{cc}
\left(\hat{\mathrm{G}}_{0 \mathbf{p}}^{r}\right)_{11}\left(\hat{\Sigma}_{1 \mathbf{p}}^{r}\right)_{11} & {\left[\left(\hat{\mathrm{G}}_{0 \mathbf{p}}^{r}\right)_{11}+\left(\hat{\mathrm{G}}_{0 \mathbf{p}}^{r}\right)_{22}\right]\left(\hat{\Sigma}_{1 \mathbf{p}}^{r}\right)_{12}} \\
{\left[\left(\hat{\mathrm{G}}_{0 \mathbf{p}}^{r}\right)_{11}+\left(\hat{\mathrm{G}}_{0 \mathbf{p}}^{r}\right)_{22}\right]\left(\hat{\Sigma}_{1 \mathbf{p}}^{r}\right)_{21}} & \left(\hat{\mathrm{G}}_{0 \mathbf{p}}^{r}\right)_{22}\left(\hat{\Sigma}_{1 \mathbf{p}}^{r}\right)_{22}
\end{array}\right)+\left(\hat{\mathrm{G}}_{0 \mathbf{p}}^{r} \rightarrow \hat{\Sigma}_{0 \mathbf{p}}^{r}, \hat{\Sigma}_{1 \mathbf{p}}^{r} \rightarrow \hat{\mathrm{G}}_{1 \mathbf{p}}^{r}\right),
$$


where $\hat{\Sigma}_{1 \mathbf{p}}^{r}=n_{i} \sum_{\mathbf{k}} \hat{T}(\mathbf{p}, \mathbf{k}) \hat{\mathrm{G}}_{1 \mathbf{k}}^{r} \hat{T}^{+}(\mathbf{p}, \mathbf{k}) . \quad \hat{I}_{r 2}^{(1)}$ vanishes due to the diagonal nature of the unperturbed retarded Green's function and self-energy. $\hat{I}_{r 3}^{(1)}$ is off-diagonal and symmetric, $\left(\hat{I}_{r 3}^{(1)}\right)_{12}=\left(\hat{I}_{r 3}^{(1)}\right)_{21}$. From this Dyson equation and the explicit forms of self-energies (B5), we find that $\hat{\mathrm{G}}_{1 \mathrm{p}}^{r}$ becomes an off-diagonal matrix with symmetric elements, $\left(\hat{\mathrm{G}}_{1 \mathbf{p}}^{r}\right)_{12}=\left(\hat{\mathrm{G}}_{1 \mathbf{p}}^{r}\right)_{21}$. Also, $\hat{\Sigma}_{1 \mathbf{p}}^{r}$ and hence $\hat{I}_{r 1}^{(1)}$ have vanishing diagonal elements. At the same time, Eq. (B7) reduces to an equation for nondiagonal elements of $\hat{\mathrm{G}}_{1 \mathbf{p}}^{r}$.

Actually, $\hat{\mathrm{G}}_{1 \mathbf{p}}^{r}$ depends on the direction of momentum through a sine function. The expression for $\hat{\mathrm{G}}_{1 \mathbf{p}}^{r}$ can be formally written as

$$
\begin{aligned}
\left(\hat{\mathrm{G}}_{1 \mathbf{p}}^{r}\right)_{\mu \bar{\mu}}= & \frac{e E \sin \phi_{\mathbf{p}}}{\omega-p^{2} / 2 m-\tau_{p}^{-1}} \\
& \times\left\{-\frac{\alpha}{2} \frac{\partial}{\partial \omega}\left[\left(\hat{\mathrm{G}}_{0 \mathbf{p}}^{r}\right)_{11}-\left(\hat{\mathrm{G}}_{0 \mathbf{p}}^{r}\right)_{22}\right]+\left[\left(\hat{\mathrm{G}}_{0 \mathbf{p}}^{r}\right)_{11}+\left(\hat{\mathrm{G}}_{0 \mathbf{p}}^{r}\right)_{22}\right] \Lambda_{p}\right\},
\end{aligned}
$$

with $\Lambda_{p}=2 n_{i} \sum_{\mathbf{k}}|V(\mathbf{p}-\mathbf{k})|^{2} \cos \left(\phi_{\mathbf{p}}-\phi_{\mathbf{k}}\right)\left(\hat{\mathcal{G}}_{1 p}^{r}\right)_{12}, \tau_{p}^{-1}=2 n_{i} \sum_{\mathbf{k}}|V(\mathbf{p}-\mathbf{k})|^{2}\left[\left(\hat{\mathrm{G}}_{0 \mathbf{p}}^{r}\right)_{11}+\left(\hat{\mathrm{G}}_{0 \mathbf{p}}^{r}\right)_{22}\right]$, and $\hat{\mathcal{G}}_{1 p}^{r}=$ $\hat{\mathrm{G}}_{1 \mathbf{p}}^{r} /\left[e E \sin \phi_{\mathbf{p}}\right]$ as a quantity independent of the direction of momentum. In the case of short-range disorders, the $\Lambda_{p}$ and $\tau_{p}$ become momentum-independent constants. From Eq. (B9) it is obvious that $\hat{\mathrm{G}}_{1 \mathrm{p}}^{r}$ does not vanish.

We note that the electron-impurity scattering is not essential for the nonvanishing of $\hat{\mathrm{G}}_{1 \mathbf{p}}^{r}$. In this, the second term on the left-hand side of Eq. (B77) plays a key role. In the absence of the electron-impurity collisions, $\hat{\mathrm{G}}_{1 \mathbf{p}}^{r}$ can be obtained analytically as

$$
\left(\hat{\mathrm{G}}_{1 \mathbf{p}}^{r}\right)_{12}=\left(\hat{\mathrm{G}}_{1 \mathbf{p}}^{r}\right)_{21}=-\frac{\alpha e E}{4\left(\omega-p^{2} / 2 m\right)} \sin \phi_{\mathbf{p}} \frac{\partial}{\partial \omega}\left[\left(\hat{\mathrm{G}}_{0 \mathbf{p}}^{r}\right)_{11}-\left(\hat{\mathrm{G}}_{0 \mathbf{p}}^{r}\right)_{22}\right] .
$$

Incidentally, from Eq. (B10) we can derive the retarded Green's function presented in our previous work 15 Using the equality $\left(\hat{\mathrm{G}}_{0 \mathbf{p}}^{r}\right) \equiv\left(\hat{\mathrm{g}}_{0 \mathbf{p}}^{r}\right)$ in the absence of electron-impurity collisions, we rewrite Eq. (B10) as $\left(\hat{\mathrm{G}}_{1 \mathbf{p}}^{r}\right)_{12}=\left(\hat{\mathrm{G}}_{1 \mathbf{p}}^{r}\right)_{21}=$ $\mathcal{A}_{1}+\mathcal{A}_{2}$ with

$$
\mathcal{A}_{1}=-\frac{e E}{4 \alpha p^{2}} \sin \phi_{\mathbf{p}}\left[\left(\hat{\mathrm{G}}_{0 \mathbf{p}}^{r}\right)_{11}-\left(\hat{\mathrm{G}}_{0 \mathbf{p}}^{r}\right)_{22}\right]
$$

and

$$
\mathcal{A}_{2}=-\frac{e E}{4 p} \sin \phi_{\mathbf{p}} \frac{\partial}{\partial \omega}\left[\left(\hat{\mathrm{G}}_{0 \mathbf{p}}^{r}\right)_{11}+\left(\hat{\mathrm{G}}_{0 \mathbf{p}}^{r}\right)_{22}\right] .
$$

$\mathcal{A}_{1}$ is just the term that we employed in Ref.15, and $\mathcal{A}_{2}$ is a total derivative with respect to $\omega$. In the absence of electron-impurity scattering, the term $\mathcal{A}_{2}$ has no effect on the spin dynamics of the considered system, because the quantity directly associated with spin-Hall current, $\hat{\mathrm{G}}_{1 \mathbf{p}}^{<}$, takes the form

$$
\hat{\mathrm{G}}_{1 \mathbf{p}}^{<}=-2 i n_{\mathrm{F}}(\omega) \operatorname{Im} \mathcal{A}_{1}+\mathcal{R},
$$

with $\mathcal{R}$ as a remainder term which can be expressed as a total derivative with respect to $\omega$ [this result is obtained from Eq. (9)].

\section{APPENDIX C: EXPLICIT EXPRESSION FOR $\hat{I}_{l}^{(1)}$}

The scattering term $\hat{I}_{l}^{(1)}$ can be expressed as the sums of $\hat{I}_{l 1}^{(1)}, \hat{I}_{l 2}^{(1)}$, and $\hat{I}_{l 3}^{(1)}$, the corresponding linear electric field parts of $\hat{I}_{l 1}, \hat{I}_{l 2}$, and $\hat{I}_{l 3}$. Further, $\hat{I}_{l 1}^{(1)}$ can be rewritten as $\hat{I}_{l 1}^{(1)}=\hat{F}_{1}+\hat{F}_{2}$, with an off-diagonal matrix $\hat{F}_{1} \equiv$ $\hat{\Sigma}_{1 \mathbf{p}}^{r} \hat{\mathrm{G}}_{0 \mathbf{p}}^{<}-\hat{\mathrm{G}}_{0 \mathbf{p}}^{<} \hat{\Sigma}_{1 \mathbf{p}}^{a}-\hat{\mathrm{G}}_{1 \mathbf{p}}^{r} \hat{\Sigma}_{0 \mathbf{p}}^{<}+\hat{\Sigma}_{0 \mathbf{p}}^{<} \hat{\mathrm{G}}_{1 \mathbf{p}}^{a}$, or explicitly,

$$
\left(\hat{F}_{1}\right)_{\mu \bar{\mu}}=\left(\hat{\Sigma}_{1 \mathbf{p}}^{r}\right)_{\mu \bar{\mu}}\left(\hat{\mathrm{G}}_{0 \mathbf{p}}^{<}\right)_{\bar{\mu} \bar{\mu}}-\left(\hat{\Sigma}_{1 \mathbf{p}}^{a}\right)_{\mu \bar{\mu}}\left(\hat{\mathrm{G}}_{0 \mathbf{p}}^{<}\right)_{\mu \mu}-\left(\hat{\mathrm{G}}_{1 \mathbf{p}}^{r}\right)_{\mu \bar{\mu}}\left(\hat{\Sigma}_{0 \mathbf{p}}^{<}\right)_{\bar{\mu} \bar{\mu}}+\left(\hat{\mathrm{G}}_{1 \mathbf{p}}^{a}\right)_{\mu \bar{\mu}}\left(\hat{\Sigma}_{0 \mathbf{p}}^{<}\right)_{\mu \mu},
$$

and a general matrix $\hat{F}_{2}$ with elements

$$
\left(\hat{F}_{2}\right)_{\mu \mu}=2 i\left[\operatorname{Im}\left(\hat{\Sigma}_{0 \mathbf{p}}^{r}\right)_{\mu \mu}\left(\hat{\mathrm{G}}_{1 \mathbf{p}}^{<}\right)_{\mu \mu}-\operatorname{Im}\left(\hat{\mathrm{G}}_{0 \mathbf{p}}^{r}\right)_{\mu \mu}\left(\hat{\Sigma}_{1 \mathbf{p}}^{<}\right)_{\mu \mu}\right],
$$




$$
\left(\hat{F}_{2}\right)_{\mu \bar{\mu}}=\left[\left(\hat{\Sigma}_{0 \mathbf{p}}^{r}\right)_{\mu \mu}-\left(\hat{\Sigma}_{0 \mathbf{p}}^{a}\right)_{\bar{\mu} \bar{\mu}}\right]\left(\hat{\mathrm{G}}_{1 \mathbf{p}}^{<}\right)_{\mu \bar{\mu}}-\left[\left(\hat{\mathrm{G}}_{0 \mathbf{p}}^{r}\right)_{\mu \mu}-\left(\hat{\mathrm{G}}_{0 \mathbf{p}}^{a}\right)_{\bar{\mu} \bar{\mu}}\right]\left(\hat{\Sigma}_{1 \mathbf{p}}^{<}\right)_{\mu \bar{\mu}},
$$

where linear electric field correction to the lesser self-energy, $\hat{\Sigma}_{1 \mathbf{p}}^{<}$, takes the form: $\hat{\Sigma}_{1 \mathbf{p}}^{<}=n_{i} \sum_{\mathbf{k}} \hat{T}(\mathbf{p}, \mathbf{k}) \hat{\mathrm{G}}_{1 \mathbf{k}}^{<} \hat{T}^{+}(\mathbf{p}, \mathbf{k})$. Furthermore, the diagonal $\hat{I}_{l 2}^{(1)}$ and off-diagonal $I_{l 3}^{(1)}$ matrices have the forms

$$
\begin{gathered}
\left(\hat{I}_{l 2}^{(1)}\right)_{\mu \mu}=i e \mathbf{E} \cdot\left(\nabla_{\mathbf{p}} \operatorname{Re}\left(\hat{\Sigma}_{0 \mathbf{p}}^{r}\right)_{\mu \mu} \frac{\partial}{\partial \omega}\left(\hat{\mathrm{G}}_{0 \mathbf{p}}^{<}\right)_{\mu \mu}-\frac{\partial}{\partial \omega} \operatorname{Re}\left(\hat{\Sigma}_{0 \mathbf{p}}^{r}\right)_{\mu \mu} \nabla_{\mathbf{p}}\left(\hat{\mathrm{G}}_{0 \mathbf{p}}^{<}\right)_{\mu \mu}\right. \\
\left.-\nabla_{\mathbf{p}} \operatorname{Re}\left(\hat{\mathrm{G}}_{0 \mathbf{p}}^{r}\right)_{\mu \mu} \frac{\partial}{\partial \omega}\left(\hat{\Sigma}_{0 \mathbf{p}}^{<}\right)_{\mu \mu}+\frac{\partial}{\partial \omega} \operatorname{Re}\left(\hat{\mathrm{G}}_{0 \mathbf{p}}^{r}\right)_{\mu \mu} \nabla_{\mathbf{p}}\left(\hat{\Sigma}_{0 \mathbf{p}}^{<}\right)_{\mu \mu}\right), \\
\left(\hat{I}_{l 3}^{(1)}\right)_{\mu \bar{\mu}}=(-1)^{\mu} \frac{e}{4} \mathbf{E} \cdot \nabla_{\mathbf{p}} \phi_{\mathbf{p}} \sum_{\nu}(-1)^{\nu}\left[\left(\hat{\mathrm{G}}_{0 \mathbf{p}}^{<}\right)_{\nu \nu} \frac{\partial}{\partial \omega} \operatorname{Re}\left(\hat{\Sigma}_{0 \mathbf{p}}^{r}\right)_{\nu \nu}-\frac{\partial}{\partial \omega}\left(\hat{\mathrm{G}}_{0 \mathbf{p}}^{<}\right)_{\nu \nu} \operatorname{Re}\left(\hat{\Sigma}_{0 \mathbf{p}}^{r}\right)_{\nu \nu}\right. \\
\left.-\frac{\partial}{\partial \omega} \operatorname{Re}\left(\hat{\mathrm{G}}_{0 \mathbf{p}}^{r}\right)_{\nu \nu}\left(\hat{\Sigma}_{0 \mathbf{p}}^{<}\right)_{\nu \nu}+\operatorname{Re}\left(\hat{\mathrm{G}}_{0 \mathbf{p}}^{r}\right)_{\nu \nu} \frac{\partial}{\partial \omega}\left(\hat{\Sigma}_{0 \mathbf{p}}^{<}\right)_{\nu \nu}\right]+\left(\hat{R}_{2}\right)_{\mu \bar{\mu}},
\end{gathered}
$$

From the definition of the lesser Green's function, we know that $\left(\hat{\mathrm{G}}_{1 \mathbf{p}}^{<}\right)_{\mu \nu}=-\left(\hat{\mathrm{G}}_{1 \mathbf{p}}^{<}\right)_{\nu \mu}^{*}$. This implies that its diagonal elements are pure imaginary-i.e., $\operatorname{Re}\left(\hat{\mathrm{G}}_{1 \mathbf{p}}^{<}\right)_{\mu \mu}=0$. Hence, we see from Eq. (B55) that the diagonal elements of the linear electric field part of the self-energy, $\Sigma_{1 \mathbf{p}}^{<}$, are also pure imaginary. Consequently, the diagonal matrix $\hat{F}_{2}$ is pure imaginary, as well as the matrix $\hat{I}_{l 2}^{(1)}$.

\section{APPENDIX D: PROOF OF $\hat{I}_{l}^{(1)}$ SUM RELATION}

First we prove Eq. (15). Since $\operatorname{Im}\left(\hat{I}_{l 1}^{(2)}\right)_{12}$ is the sum of $\left(\hat{F}_{1}\right)_{12}$ and $\operatorname{Im}\left(\hat{F}_{2}\right)_{12}$, we first consider the integral $\mathcal{I}_{1} \equiv$ $\sum_{\mathbf{p}} \sin \phi_{\mathbf{p}}\left(\hat{F}_{1}\right)_{12}$. Explicitly, it takes the form

$$
\mathcal{I}_{1}=\sum_{\mathbf{p}} \sin \phi_{\mathbf{p}}\left\{\operatorname{Re}\left(\hat{\Sigma}_{1 \mathbf{p}}^{r}\right)_{12}\left[\operatorname{Im}\left(\hat{\mathrm{G}}_{0 \mathbf{p}}^{<}\right)_{22}-\operatorname{Im}\left(\hat{\mathrm{G}}_{0 \mathbf{p}}^{<}\right)_{11}\right]-\operatorname{Re}\left(\hat{\mathrm{G}}_{1 \mathbf{p}}^{r}\right)_{12}\left[\operatorname{Im}\left(\hat{\Sigma}_{0 \mathbf{p}}^{<}\right)_{22}-\operatorname{Im}\left(\hat{\Sigma}_{0 \mathbf{p}}^{<}\right)_{11}\right]\right\} .
$$

Substituting the self-energies into Eq. (D1), we have

$$
\begin{aligned}
\mathcal{I}_{1}= & \sum_{\mathbf{p k}} \sin \phi_{\mathbf{p}}|V(\mathbf{p}-\mathbf{k})|^{2}\left\{\operatorname{Re}\left(\hat{\mathrm{G}}_{1 \mathbf{k}}^{r}\right)_{12}\left[\operatorname{Im}\left(\hat{\mathrm{G}}_{0 \mathbf{p}}^{<}\right)_{22}-\operatorname{Im}\left(\hat{\mathrm{G}}_{0 \mathbf{p}}^{<}\right)_{11}\right]\right. \\
& \left.+\cos \left(\phi_{\mathbf{p}}-\phi_{\mathbf{k}}\right) \operatorname{Re}\left(\hat{\mathrm{G}}_{1 \mathbf{p}}^{r}\right)_{12}\left[\operatorname{Im}\left(\hat{\mathrm{G}}_{0 \mathbf{k}}^{<}\right)_{11}-\operatorname{Im}\left(\hat{\mathrm{G}}_{0 \mathbf{k}}^{<}\right)_{22}\right]\right\} .
\end{aligned}
$$

Interchanging the dummy integration variables $\mathbf{p}$ and $\mathbf{k}, \mathcal{I}_{1}$ can be rewritten as

$$
\begin{aligned}
\mathcal{I}_{1} & =\sum_{\mathbf{p k}}|V(|\mathbf{p}-\mathbf{k}|)|^{2}\left(\sin \phi_{\mathbf{p}}-\sin \phi_{\mathbf{k}} \cos \left(\phi_{\mathbf{p}}-\phi_{\mathbf{k}}\right)\right) \operatorname{Re}\left(\hat{\mathrm{G}}_{1 \mathbf{k}}^{r}\right)_{12}\left[\operatorname{Im}\left(\hat{\mathrm{G}}_{0 \mathbf{p}}^{<}\right)_{22}-\operatorname{Im}\left(\hat{\mathrm{G}}_{0 \mathbf{p}}^{<}\right)_{11}\right] \\
& =\sum_{\mathbf{p k}}|V(|\mathbf{p}-\mathbf{k}|)|^{2} \cos \phi_{\mathbf{k}} \sin \left(\phi_{\mathbf{p}}-\phi_{\mathbf{k}}\right) \operatorname{Re}\left(\hat{\mathrm{G}}_{1 \mathbf{k}}^{r}\right)_{12}\left[\operatorname{Im}\left(\hat{\mathrm{G}}_{0 \mathbf{p}}^{<}\right)_{22}-\operatorname{Im}\left(\hat{\mathrm{G}}_{0 \mathbf{p}}^{<}\right)_{11}\right] .
\end{aligned}
$$

From relation (B6) it can be seen that $\mathcal{I}_{1}=0$.

Next, we analyze the remaining component of the integral, $\mathcal{I}_{2} \equiv \sum_{\mathbf{p}} \sin \phi_{\mathbf{p}} \operatorname{Im}\left(\hat{F}_{2}\right)_{12}$,

$$
\begin{aligned}
\mathcal{I}_{2}= & \sum_{\mathbf{p}} \sin \phi_{\mathbf{p}}\left\{\operatorname{Re}\left(\hat{\mathrm{G}}_{1 \mathbf{p}}^{<}\right)_{12}\left[\operatorname{Im}\left(\Sigma_{0 \mathbf{p}}^{r}\right)_{11}+\operatorname{Im}\left(\Sigma_{0 \mathbf{p}}^{r}\right)_{22}\right]\right. \\
& \left.+\operatorname{Im}\left(\hat{\mathrm{G}}_{1 \mathbf{p}}^{<}\right)_{12}\left[\operatorname{Re}\left(\Sigma_{0 \mathbf{p}}^{r}\right)_{11}+\operatorname{Re}\left(\Sigma_{0 \mathbf{p}}^{r}\right)_{22}\right]-(\hat{\Sigma} \leftrightarrow \hat{\mathrm{G}})\right\} .
\end{aligned}
$$

Again substituting the self-energies into Eq. (D4), this integral can be simplified as

$$
\begin{aligned}
\mathcal{I}_{2}= & \sum_{\mathbf{p}, \mathbf{k}}|V(\mathbf{p}-\mathbf{k})|^{2} \cos \phi_{\mathbf{k}} \sin \left(\phi_{\mathbf{p}}-\phi_{\mathbf{k}}\right) \operatorname{Re}\left(\hat{\mathrm{G}}_{1 \mathbf{p}}^{<}\right)_{12}\left[\operatorname{Im}\left(\hat{\mathrm{G}}_{0 \mathbf{k}}^{r}\right)_{11}+\operatorname{Im}\left(\hat{\mathrm{G}}_{0 \mathbf{k}}^{r}\right)_{22}\right] \\
& -\frac{1}{2} \sum_{\mathbf{p}, \mathbf{k}}\left[\cos \phi_{\mathbf{k}}-\cos \phi_{\mathbf{p}} \cos \left(\phi_{\mathbf{p}}-\phi_{\mathbf{k}}\right)\right]\left[\operatorname{Im}\left(\hat{\mathrm{G}}_{0 \mathbf{p}}^{r}\right)_{11}+\operatorname{Im}\left(\hat{\mathrm{G}}_{0 \mathbf{p}}^{r}\right)_{22}\right]\left[\operatorname{Im}\left(\hat{\mathrm{G}}_{1 \mathbf{k}}^{<}\right)_{11}-\operatorname{Im}\left(\hat{\mathrm{G}}_{1 \mathbf{k}}^{<}\right)_{22}\right] .
\end{aligned}
$$


Here, we have used Eq. (BB6). In this, the vanishing of the real parts of the diagonal elements of the distribution function-i.e., $\operatorname{Re}\left(\hat{\mathrm{G}}_{1 \mathbf{k}}^{<}\right)_{\mu \mu}=0$-has also been considered. Combining the terms proportional to $\cos \phi_{\mathbf{p}} \cos \left(\phi_{\mathbf{p}}-\phi_{\mathbf{k}}\right)$ in the second line of Eq. (D5) with a similar term in the first line, we obtain

$$
\begin{aligned}
\mathcal{I}_{2}= & \frac{1}{2} \sum_{\mathbf{p}} \cos \phi_{\mathbf{p}}\left\{\left[\operatorname{Im}\left(\hat{\Sigma}_{1 \mathbf{p}}^{<}\right)_{11}-\operatorname{Im}\left(\hat{\Sigma}_{1 \mathbf{p}}^{<}\right)_{22}\right]\left[\operatorname{Im}\left(\hat{\mathrm{G}}_{0 \mathbf{p}}^{r}\right)_{11}+\operatorname{Im}\left(\hat{\mathrm{G}}_{0 \mathbf{p}}^{r}\right)_{22}\right]\right. \\
& \left.-\left[\operatorname{Im}\left(\hat{\Sigma}_{0 \mathbf{p}}^{<}\right)_{11}+\operatorname{Im}\left(\hat{\Sigma}_{0 \mathbf{p}}^{<}\right)_{22}\right]\left[\operatorname{Im}\left(\hat{\mathrm{G}}_{1 \mathbf{p}}^{r}\right)_{11}-\operatorname{Im}\left(\hat{\mathrm{G}}_{1 \mathbf{p}}^{r}\right)_{22}\right]\right\} .
\end{aligned}
$$

Further, we note that in the self-consistent approximation, there is a vanishing quantity $\mathcal{K}$ given by

$$
\mathcal{K} \equiv \sum_{\mathbf{p}} \cos \phi_{\mathbf{p}}\left\{\left[\operatorname{Im}\left(\hat{\mathrm{G}}_{0 \mathbf{p}}^{r}\right)_{11}-\operatorname{Im}\left(\hat{\mathrm{G}}_{0 \mathbf{p}}^{r}\right)_{22}\right]\left[\operatorname{Im}\left(\hat{\Sigma}_{1 \mathbf{p}}^{<}\right)_{11}+\operatorname{Im}\left(\hat{\Sigma}_{1 \mathbf{p}}^{<}\right)_{22}\right]-(\Sigma \leftrightarrow \mathrm{G})\right\} .
$$

The vanishing of $\mathcal{K}=0$ can be shown by inserting the explicit forms of the self-energies, Eq. (B5), into $\mathcal{K}$ and employing Eq. (B6). Finally, adding $\mathcal{K}$ to the right hand side of Eq. (D6), we find

$$
\begin{aligned}
\mathcal{I}_{2} & =\frac{1}{2} \sum_{\mathbf{p} \mu} \cos \phi_{\mathbf{p}}(-1)^{\mu+1}\left\{\operatorname{Im}\left(\hat{\Sigma}_{1 \mathbf{p}}^{<}\right)_{\mu \mu} \operatorname{Im}\left(\hat{\mathrm{G}}_{0 \mathbf{p}}^{r}\right)_{\mu \mu}-\operatorname{Im}\left(\hat{\Sigma}_{0 \mathbf{p}}^{<}\right)_{\mu \mu} \operatorname{Im}\left(\hat{\mathrm{G}}_{1 \mathbf{p}}^{r}\right)_{\mu \mu}\right\} \\
& =\sum_{\mathbf{p}} \frac{\cos \phi_{\mathbf{p}}}{2}\left[\operatorname{Re}\left(\hat{F}_{2}\right)_{11}-\operatorname{Re}\left(\hat{F}_{2}\right)_{22}\right]
\end{aligned}
$$

which is Eq. 15.

In the following, we prove Eq. (16). From Eq. [C5), the involved integral, denoted as $\mathcal{I}_{3}$, can be written as

$$
\begin{aligned}
\mathcal{I}_{3}= & \frac{i}{4} \sum_{\mathbf{p} \mu \nu} \int \frac{d \omega}{2 \pi}(-1)^{\nu} \cos \phi_{\mathbf{p}} e \mathbf{E} \cdot \nabla_{\mathbf{p}}\left\{\left(\hat{\mathrm{G}}_{0}^{<}\right)_{\nu \nu} \frac{\partial}{\partial \omega} \operatorname{Re}\left(\hat{\Sigma}_{0}^{r}\right)_{\nu \nu}-\left[\frac{\partial}{\partial \omega}\left(\hat{\mathrm{G}}_{0}^{<}\right)_{\nu \nu}\right] \operatorname{Re}\left(\hat{\Sigma}_{0}^{r}\right)_{\nu \nu}\right. \\
& \left.-\left[\frac{\partial}{\partial \omega} \operatorname{Re}\left(\hat{\mathrm{G}}_{0}^{r}\right)_{\nu \nu}\right]\left(\hat{\Sigma}_{0}^{<}\right)_{\nu \nu}+\operatorname{Re}\left(\hat{\mathrm{G}}_{0}^{r}\right)_{\nu \nu} \frac{\partial}{\partial \omega}\left(\hat{\Sigma}_{0}^{<}\right)_{\nu \nu}\right] .
\end{aligned}
$$

Here, the momentum integral of Eq. (C5) has been performed by parts integration, and the contribution from $\left(\hat{R}_{2}\right)_{12}$ vanishes due to its total derivative nature with respect to $\omega$. Each part of the integrand involves two functions $\hat{\mathrm{G}}_{0}^{r,<}$ and $\hat{\Sigma}_{0}^{r,<}$. Further integration by parts with respect to $\omega$ in terms of the form $\left(\frac{\partial}{\partial \omega} \nabla_{\mathbf{p}} \mathrm{A}\right.$ ) B ([with A and B being $\left(\hat{\mathrm{G}}_{0}^{r,<}\right)_{\mu \mu}$ and $\left(\hat{\Sigma}_{0}^{r,<}\right)_{\nu \nu}$, respectively, or vice versa] results in the operators $\nabla_{\mathbf{p}}$ and $\partial / \partial \omega$ acting individually on $\mathrm{A}$ or B-i.e., $\left(\frac{\partial}{\partial \omega} \mathrm{A}\right) \nabla_{\mathbf{p}}$ B. Thus, we obtain

$$
\begin{aligned}
\mathcal{I}_{3}= & \frac{i}{2} \sum_{\mathbf{p} \mu \nu} \int \frac{d \omega}{2 \pi}(-1)^{\nu} \cos \phi_{\mathbf{p}} e \mathbf{E} \cdot\left[\nabla_{\mathbf{p}}\left(\hat{\mathrm{G}}_{0}^{<}\right)_{\nu \nu} \frac{\partial}{\partial \omega} \operatorname{Re}\left(\hat{\Sigma}_{0}^{r}\right)_{\nu \nu}-\frac{\partial}{\partial \omega}\left(\hat{\mathrm{G}}_{0}^{<}\right)_{\nu \nu} \nabla_{\mathbf{p}} \operatorname{Re}\left(\hat{\Sigma}_{0}^{r}\right)_{\nu \nu}\right. \\
& \left.-\frac{\partial}{\partial \omega} \operatorname{Re}\left(\hat{\mathrm{G}}_{0}^{r}\right)_{\nu \nu} \nabla_{\mathbf{p}}\left(\hat{\Sigma}_{0}^{<}\right)_{\nu \nu}+\nabla_{\mathbf{p}} \operatorname{Re}\left(\hat{\mathrm{G}}_{0}^{r}\right)_{\nu \nu} \frac{\partial}{\partial \omega}\left(\hat{\Sigma}_{0}^{<}\right)_{\nu \nu}\right] .
\end{aligned}
$$

Using Eq. (C4), Eq. (16) can be derived immediately.

* Electronic address: liusy@mail.sjtu.edu.cn

1 M. I. Dyakonov and V. I. Perel, Phys. Lett. 35A, 459 (1971).

2 J. E. Hirsch, Phys. Rev. Lett. 83, 1834 (1999).

3 S. Murakami, N. Nagaosa, and S. C. Zhang, Science 301, 1348 (2003).

4 J. Sinova, D. Culcer, Q. Niu, N. A. Sinitsyn, T. Jungwirth, and A. H. MacDonald, Phys. Rev. Lett. 92, 126603 (2004).

5 Y. K. Kato, R. C. Myers, A. C. Gossard, and D. D. Awschalom, Science 306, 1910 (2004).

6 J. Wunderlich, B. Kaestner, J. Sinova, and T. Jungwirth, Phys. Rev. Lett. 94, 047204 (2005).

7 J. Schliemann and D. Loss, Phys. Rev. B 69, 165315 (2004).

8 K. Nomura, J. Sinova, T. Jungwirth, Q. Niu, and A. H. MacDonald, Phys. Rev. B 71, 041304(R) (2005).

9 O. Chalaev and D. Loss, Phys. Rev. B 71, 245318 (2005).

10 O. V. Dimitrova, cond-mat/0405339 (unpublished). 
11 J. I. Inoue, G. E. W. Bauer, and L. W. Molenkamp, Phys. Rev. B 70, 041303(R) (2004).

12 R. Raimondi and P. Schwab, Phys. Rev. B 71, 033311 (2005).

13 A. Khaetskii, cond-mat/0408136 (unpublished).

14 E. G. Mishchenko, A. V. Shytov, and B. I. Halperin, Phys. Rev. Lett. 93, 226602 (2004).

15 S. Y. Liu and X. L. Lei, cond-mat/0411629 (unpublished).

16 N. Sugimoto, S. Onoda, S. Murakami, and N. Nagaosa, cond-mat/0503475 (unpublished).

17 N. J. Horing, in Proceedings of the International Conference on the Physics Semiconductors Exeter, 1962, (Institute of Physics and Physical Society, London, 1962) P. 58, and references cited therein.

18 F. S. Khan, J. H. Davies, and J. W. Wilkins, Phys. Rev. B 36, 2578 (1987).

19 L. Reggiani, P. Lugli, and A. P. Jauho, Phys. Rev. B 36, 6602 (1987).

20 E. I. Rashba, Fiz. Tverd. Tela (Leningrad) 2, 1224 (1960) [Sov. Phys. Solid State 2, 1109 (1960)]; Y. A. Bychkov and E. I. Rashba, J. Phys. C 17, 6039 (1984).

21 G. D. Mahan, Many-Particle Physics (Plenum, New York, 1990).

${ }^{22}$ H. Haug and A.-P. Jauho, Quantum Kinetics in Transport and Optics of Semiconductors (Springer, 1996).

23 S. Y. Liu and X. L. Lei, Phys. Rev. B 72, 195329 (2005).

${ }^{24}$ R. Kubo, J. Phys. Soc. Japan 12, 570 (1957); P.C. Martin and J. Schwinger, Phys. Rev. 115, 1342 (1959).

25 P.-Q. Jin, Y.-Q. Li, and F.-C. Zhang, cond-mat/0502231 (unpublished).

26 S. Murakami, N. Nagaosa, and S.-C. Zhang, Phys. Rev. B 69, 235206 (2004).

27 S. Zhang and Z. Yang, Phys. Rev. Lett. 94, 066602 (2005).

28 P. Zhang, J. Shi, D. Xiao, and Q. Niu, cond-mat/0503505 (unpublished).

29 P.L. Krotkov and S. Das Sarma, cond-mat/0510114 (unpublished).

30 See, for example, M. Q. Weng and M. W. Wu, Phys. Rev. B 66, 235109 (2002); J. Appl. Phys. 93, 410 (2003). 